\title{
A rare case of stroke mimic in a young patient
}

J. Talbot ${ }^{1}$, O. Almasri', E. Abdelgadir ${ }^{1}$, J. Newman ${ }^{1}$, A. Shivane ${ }^{2}$, D.A. Hilton ${ }^{2}$, A. Mohd Nor ${ }^{1}$. University Hospitals Plymouth NHS Trust, Neurology ${ }^{1}$ and Neuropathology ${ }^{2}$, Plymouth, United Kingdom.

\section{Introduction}

We report a rare cause of a stroke mimic due to intravascular large $B$ cell lymphoma (ILBCL). ILBCL is a very rare and aggressive form of non-Hodgkin lymphoma with a predilection for blood vessel walls and skin. Diagnosis is extremely challenging and often made post-mortem.

\section{Case report}

A 45 year-old Caucasian Jehovah's witness with a background of depression was referred with a 2-3 day history of speech decline, left weakness, right arm spasm, and altered behaviour. She was unable to verbalise and had an upgoing right plantar on examination. CT head in the Emergency department showed a right cerebellar infarct. Subsequent MRI revealed multiple areas of restricted diffusion in keeping with multi-territory infarcts (Fig 1). Extensive tests including young stroke panel failed to reveal a cause for her stroke (Tab 1).

Her condition progressed with further left-sided weakness and focal seizures. Repeat imaging showed radiological progression (Fig 2). In addition to antiplatelet and statin treatment she received pulsed steroids, but with limited clinical improvement. 34 days after admission she deteriorated with reduction in her conscious level, pyrexia and haemolytic anaemia. Repeat imaging showed intraparenchymal haemorrhage and she died shortly after. Post mortem examination showed ILBCL throughout (Fig 3).
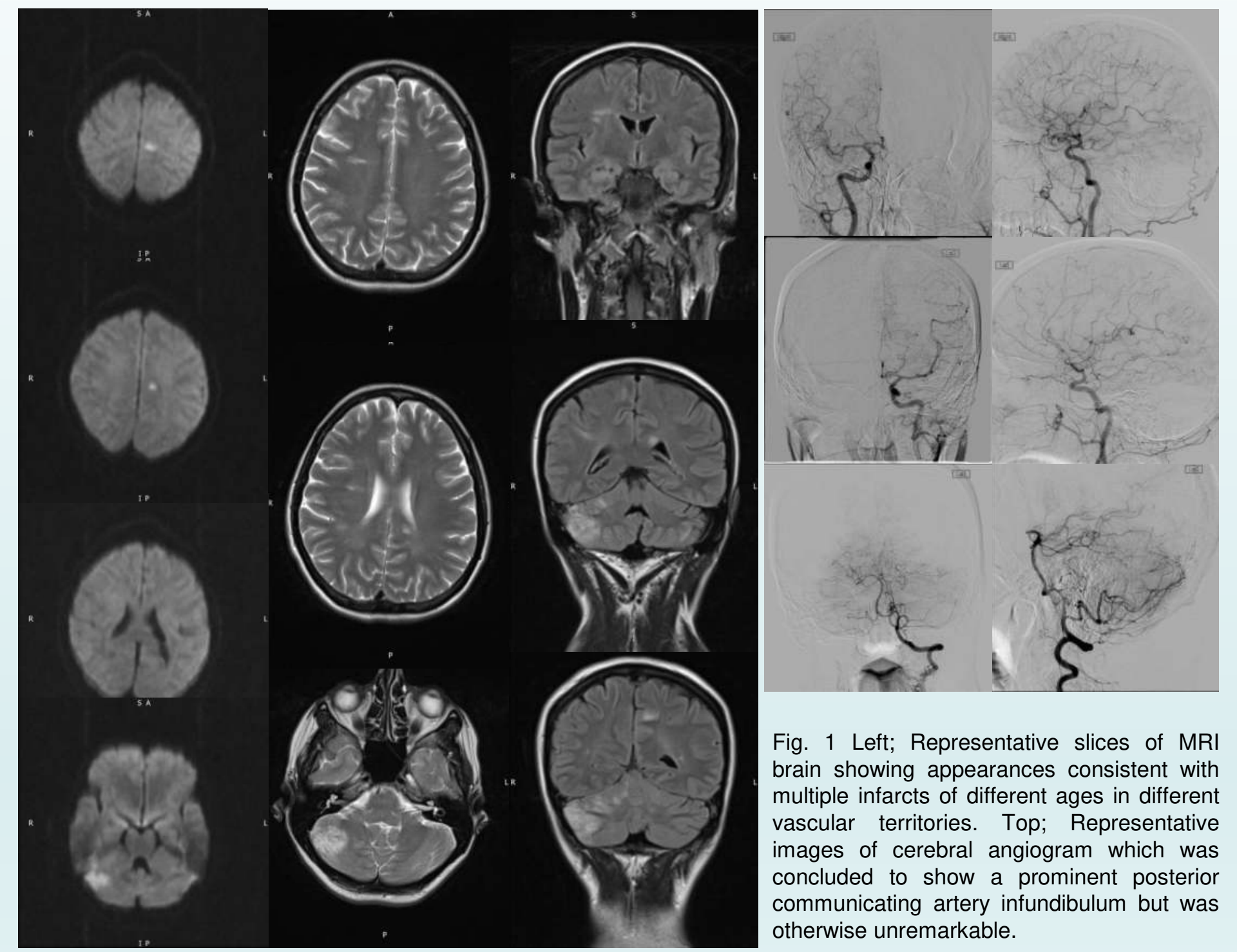

Fig. 1 Left; Representative slices of MRI brain showing appearances consistent with multiple infarcts of different ages in different vascular territories. Top; Representative mages of cerebral angiogram which was concluded to show a prominent posterio communicating artery infundibulum but was otherwise unremarkable.

\begin{tabular}{l|l} 
Relevant Test & Result \\
\hline FBC & Normocytic anaemia at 115g/L \\
\hline Plasma viscosity & Normal \\
\hline LDH & Raised at 400 iu/L, improving on repeat testing \\
\hline Blood film & Unremarkable \\
\hline CSF analysis & Glucose 3.0mmol/L, Protein 0.61 g/L, Lymphocytes 2, RBC 37 \\
\hline CSF analysis repeat & Glucose 2.6, Protein 0.54, Lymphocytes <1. RBC 1, Cytology - no cells \\
\hline Direct Coomb's test & Positive \\
\hline CT Thorax, Abdomen, Pelvis & No definite evidence of malignancy or lymphadenopathy
\end{tabular}

Tab 1 Relevant investigation during the course of admission.

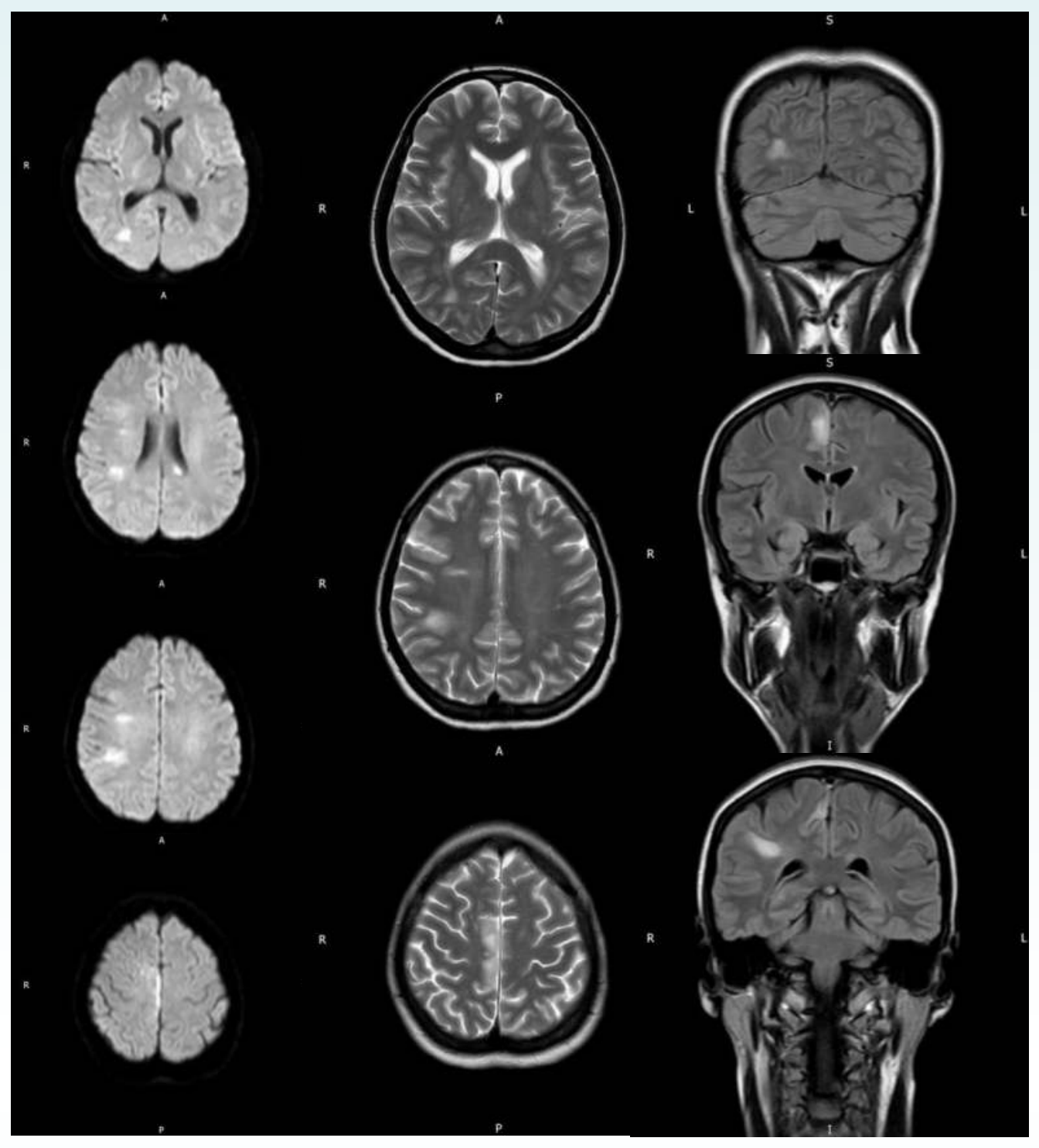

Fig. 2 Representative slices of repeat MRI brain showing progression of the previously noted lesion and further areas of restricted diffusion. Not shown; contrast enhancement in the cerebellar, right medial frontal and parietal lesions.

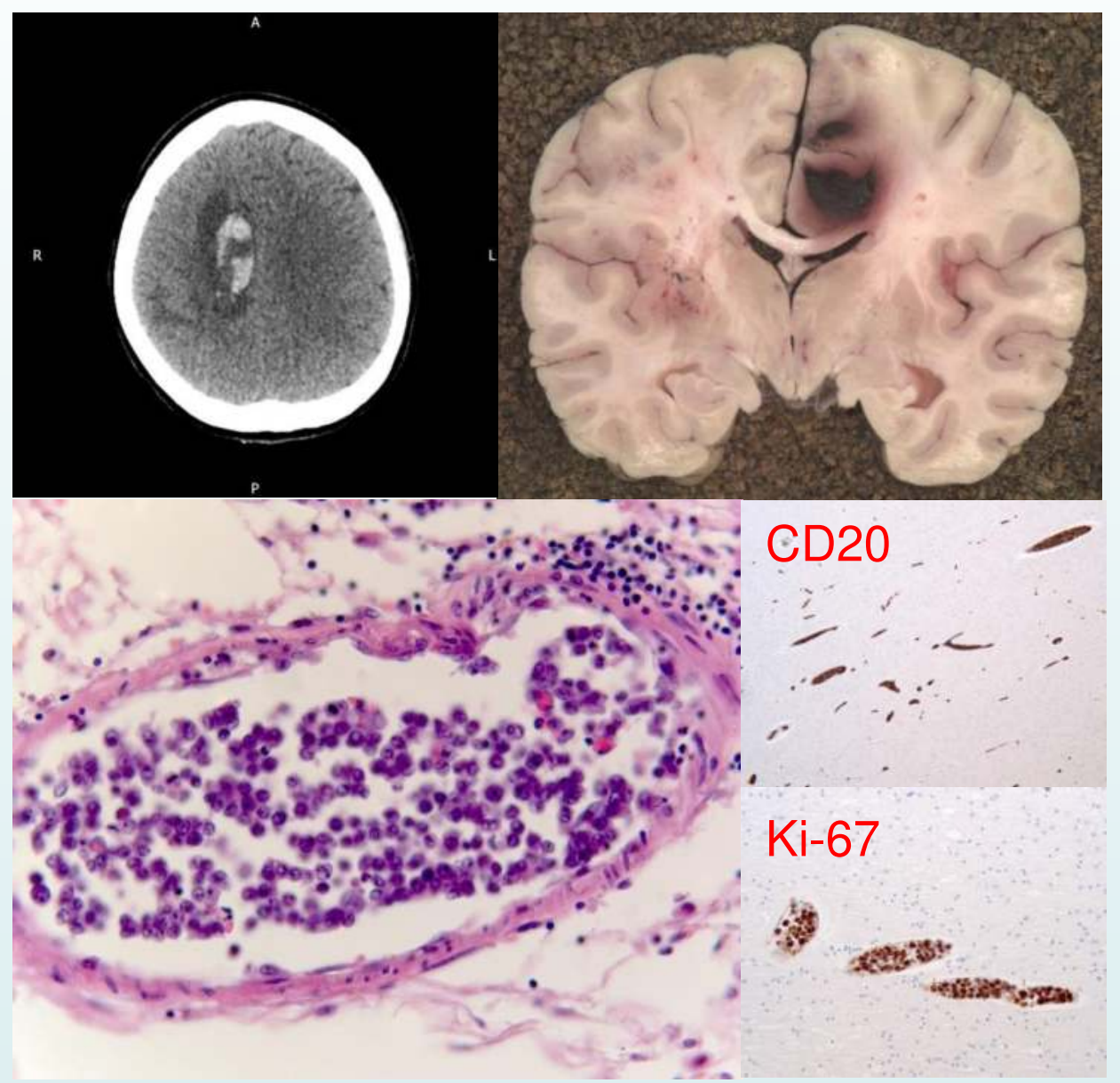

Fig. 3 Top left; CT head showing new intraparenchymal haemorrhage. Top right gross pathology showing mild generalised brain swelling and an area of acute haemorrhage in the right superior medial hemisphere. Botton; Histology confirming intravascular malignant lymphoid cells (left) positive with B-cell marker CD20 and showing increased proliferation in a Ki-67 stain

\section{Discussion}

ILBCL is a rare cause of stroke. The true incidence remains elusive. A diagnosis of ILBCL should be considered in patients with multi-territory infarcts without a clear cause (e.g. vasculitis, atrial fibrillation). Presentation is thought to vary between the Western and Asian populations. The former tend to present with neurological or dermatological symptoms, whereas the latter can present with multi-organ failure, pancytopenia, fever and bone marrow involvement. Diagnosis can be achieved through skin or other affected organ (including bone marrow) biopsy during life, or on autopsy. Due to frequent CNS involvement, evaluation often includes MRI brain and CSF analysis. Anthracycline-based chemotherapy has been used in the treatment of ILBCL with 3-year survival rate reported at $33 \%$, and this may be improved with adjunct Rituximab therapy. 